\title{
Rotation rates of pre-main sequence stars: role of circumstellar disk
}

\author{
Somnath Dutta ${ }^{1,2 *}$,Soumen Mondal ${ }^{2 \dagger}$, Santosh Joshi ${ }^{3}$, Ramkrishna Das $^{2}$ \\ ${ }^{1}$ Indian Institute of Science Education and Research Tirupati, Tirupati -517 507, India \\ ${ }^{2}$ S. N. Bose National Centre for Basic Sciences, Kolkata-700 106, India \\ ${ }^{3}$ Aryabhatta Research Institute of Observational Sciences (ARIES), Manora Peak, \\ Nainital-263 002, India
}

\begin{abstract}
Photometric variability with new high-cadence, long-duration data has drawn a huge interest to explore the time domain behaviour of young stellar objects since the last few decades. Here we provide an overview of optical photometric observations of the young clusters, identification of variable nature from their light curves, characterization of their circumstellar disk. Based on our studies, we find that $50-65 \%$ of young stars are variable in our studied regions. Most of the periodic young stars have periods less than 10 days. Diskbearing stars have relatively larger amplitudes than the older population, which indicate that the young stellar variability could be due to the effect of the hotspot, asymmetric disk obscuration, accretion outbursts or any other structural modification in the inner disk regions.
\end{abstract}

Keywords: young stars - optical observations - photometric variability - periodic variables - circumstellar material

\section{Introduction}

After the pioneering discovery of Joy (1945), variability has been a well-known phenomenon of premain sequence (PMS) stars. Stellar variability could be intimately linked with the disk evolution and accretion (Romanova et al. 2004, Turner et al. 2010), therefore it can provide insight about the early evolution of stars. There are various mechanisms that drive stellar variability. Young stars are believed to consist of the highly active chromosphere, coronae (e.g., Costa et al., 2000) with extensive star spots (Herbst et al., 2007) and optical flaring (Kowalski et al., 2010) associated with sun-like magnetic activity. Most importantly, the strong magnetic field of young stars channelizes the material accretion from disk to star surface onto a particular location along the field lines, resulting in an accretion shock of temperature $\sim 10^{6} \mathrm{~K}$ and a post-shock "hotspot" of $\sim 10^{4} \mathrm{~K}$. In the case of a steady accretion flow, the star will appear to vary periodically due to hotspot rotation (Mahdavi \& Kenyon 1998). Moreover, the star is not corotating with the inner circumstellar disk, thus magnetic field lines connecting the star and disk will be stretched and distorted, eventually reconnecting with the rotation of disk and star.

\footnotetext{
*duttasomnath9@gmail.com

†soumen.mondal@bose.res.in
} 
These reconnection events may produce more powerful flares than ordinary coronal flares (Favata et al., 2005) and contribute to the flux variation. Asymmetric circumstellar disk turbulence is able to produce transient dust structures away from the plane of the disk, which may intersect our line-ofsight to produce luminosity variation (Turner et al. 2010). At the very early stages of star formation, the circumstellar disk may grow massive enough accreting from its surrounding envelope and become gravitationally unstable to fragment (Vorobyov \& Basu 2010). When individual fragments fall onto the star, the luminosity will change by a few orders of magnitudes, possibly the case observed in FU-Ori events.

All such different mechanisms could create rare, unique variable phenomena on their time domain light curves, which are closely related to circumstellar material. However, optical time-series studies of young stars are very challenging due to their circumstellar material. One way to explore them is deep optical imaging in $I$-band, which can probe the young stars better than other bluer optical bands (e.g., $U, B, V$ ). Another challenging fact is that the variability studies require a long-time series observations up to years, making itself difficult in the regime of limited time-schedule observations. Since last few decades several authors have attempted to probe the variability in young stars from an observational perspective, and to establish the importance of accretion and disk evolution in variability (Herbst et al. 1994; Rebull et al. 2006; Herbst et al. 2007; Irwin et al. 2008; Cieza \& Baliber 2007).

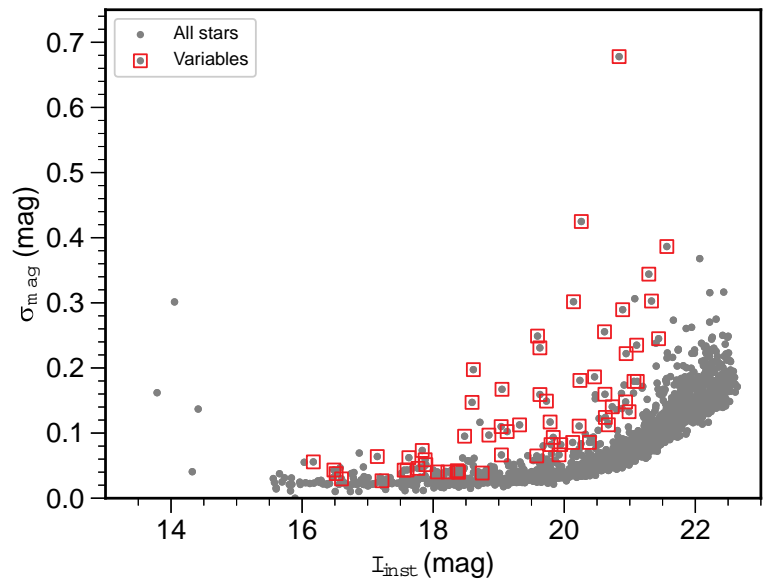

Figure 1: The rms of all the observed stars from our time-series analyses is shown in grey dots for a the cluster NGC 2282. The red squares are the candidate variables identified from our analyses.

In this paper, we present our studies of time-series observations on $I$-band on some young clusters e.g. NGC 2282, Cyg OB7 and NGC 2264. NGC $2282\left(\alpha_{2000}=06^{h} 46^{m} 50.4^{s} \delta_{2000}=+01^{0} 18^{m} 50^{s}\right)$ is a cluster embedded in the Monoceros constellation (Lada \& Lada 2003). NGC 2282 contains more than 150 disk-bearing stars within a small $(\sim 3.2 \mathrm{arcmin})$ and relatively nearby $(\sim 1.65 \mathrm{kpc})$ region (Dutta et al. 2015), thus making it a suitable target to explore the young variable stars. Another target Cygnus OB7 (Cyg OB7; Reipurth \& Schneider 2008, Dutta et al. 2019) is the nearest ( 760 pc) of all Cygnus OB association. We observed this region as a part of our young stellar variability search. We are also monitoring the young cluster NGC $2264\left(\alpha_{2000}=06^{h} 40^{m} 58^{s} \delta_{2000}=+09^{0} 53^{m} 45^{s}\right)$, which is located at $\sim 760 \mathrm{pc}$ and contains more than 1000 members (Dahm 2008). Thus, NGC 2264 would provide us with a good opportunity to probe young stellar variability. However, for simplicity, we have mostly presented our results from NGC 2282. We have structured the paper as follows. In section 2, we have discussed the techniques of our variability studies with various optical telescopes. Light curve generation, identification of variables from their light curves and period measurements are demonstrated in section 3 . 


\section{Observational techniques}

We have observed/are observing those targets in optical $I$-band with various optical telescopes, such as 1.3-m Devasthal fast Optical Telescope (DFOT), ARIES, Nainital, India; 2-m Himalayan Chandra Telescope (HCT), India; 1.04-m Sampurnanad Telescope (ST), ARIES. The observations are performed with suitable binning mode (e.g. $2 \times 2$ ) to get a good signal-to-noise ratio (SNR). We prefer to observe in different exposure time (e.g., $30 \mathrm{~s}, 60 \mathrm{~s}, 120 \mathrm{~s}, 180 \mathrm{~s}$ exposure for each frame) to obtain a good dynamic coverage of bright as well as faint objects. Detailed telescope specifications and observational techniques could be found in Dutta et al. (2018a). The main challenge when using various telescopes simultaneously is the systematic offset, which we need to adjust for the different observing facilities. One optimal way is to find the linear difference between the non-variable sources observed in difference telescopes, which is to be added as an offset for all other sources.

Deep near-infrared (NIR) observations around our targets are obtained in $H(1.63 \mu \mathrm{m})$ and $K$ $(2.14 \mu \mathrm{m})$ bands from the CFHT data archive $^{1}$ built with observations from the WIRCam camera of the CFHT 3.6m telescope. We also utilized the UKIDSS Galactic Plane Survey (Lucas et al. 2008) depending upon availability in the archive. The Spitzer-IRAC observations in the 3.6 and $4.5 \mu \mathrm{m}$ bands were available in the Spitzer archive ${ }^{2}$. We made use of INT Photometric H $\alpha$ Survey (IPHAS ${ }^{3}$ ), which is a photometry survey in the Northern Milky Way in visible light $(\mathrm{H} \alpha, \mathrm{r}, \mathrm{i})$ using the wide-field camera on the 2.5-m Isaac Newton Telescope (INT) in La Palma (Barentsen et al. 2014).

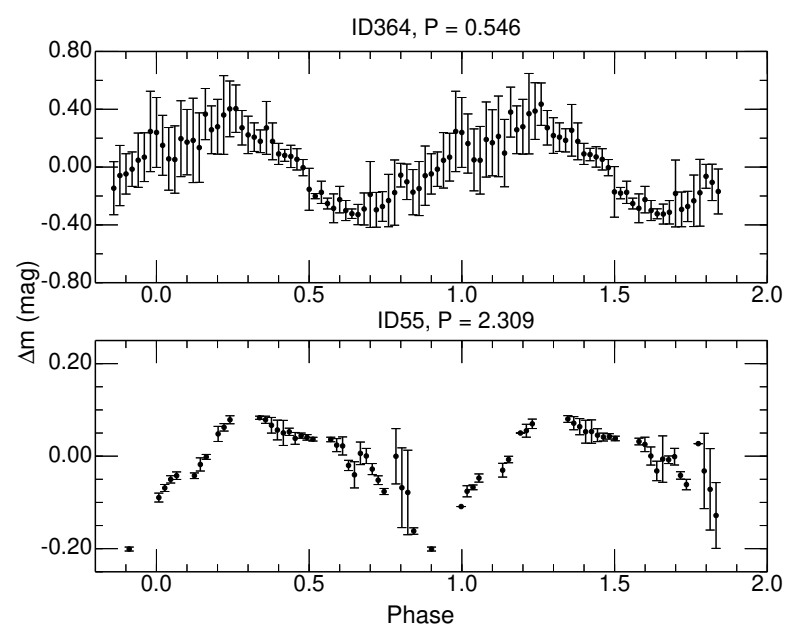

Figure 2: Phase light curves of two variable stars from NGC 2282.

\section{Analyses}

\subsection{Identification of variable stars}

\subsubsection{Relative magnitude light curves}

The identification of variable stars with ground-based telescopes depends significantly on the sky background variation within the same night or from night-to-night. To reduce this effect, one widely used technique is the relative magnitude measurement with respect to a secondary standard (Dutta

\footnotetext{
${ }^{1}$ http://www.cadc-ccda.hia-iha.nrc-cnrc.gc.ca/en/cfht/

${ }^{2} \mathrm{http} / / /$ archive.spitzer.caltech.edu/

${ }^{3}$ http://www.iphas.org/data.shtml
} 
et al. 2018a, and references therein). For this, we need a set of non-variable sources with different magnitude ranges in the target field, which should be taken from the same frame as the variable stars. Our variable star detection relies on the proper selection of non-variable sources. From the careful inspection of the the root-mean-square ( $\mathrm{rms}$ ) variation, light curves (on observed magnitude scale), phase light curves (folding with periods), we have extracted 10 optimal non-variable sources with magnitudes ranging from 13-19 for further analyses. We have then averaged all 10 non-variable magnitudes from each frame to achieve the final reference magnitude $\left(M_{\text {ref }} s\right)$ over the whole observation span. $M_{r e f} \mathrm{~s}$ are then subtracted from all remaining stars to obtain the light curves.

\subsubsection{Scattering of magnitudes from normal trend}

Since there are more than one thousand sources towards our target field, a variability diagnostic should be introduced to avoid visual inspection of all light curves. For simplicity, we have adopted the rms $\left(\sigma_{m a g}\right)$ variation of the data points, which suits well to our single band ( $I$-band) time-series observations. As an example, we have displayed the $r m s$ as a function of magnitude in Figure 1 for the sample from Dutta et al. (2018a) of our NGC 2282 observations. The figure shows the exponentially increasing trend of $r m s$ with magnitude, which basically demonstrates that the SNR is decreasing with the faint magnitudes.

However, several targets stand above the general trend and deserve further scrutiny. Such outliers result either from intrinsic variability or bad CCD pixels, cosmic ray events, saturated pixels (especially in the brighter end). First, we select all the sources with $3 \sigma$ deviation in each magnitude bin. Then, we perform visual inspection light curves (see section 3.1.1) to search for any known variability pattern or other systematic variation. In a few cases, we realize that the large sigma is only due to one or a few outlying data points, and so that this target is not to be considered as a variable star. Such objects are removed from the variable list.

In this approach, we definitely miss some of the smallest-amplitude variables. We believed that those low-amplitude variables are mostly contributed by binary or other non-PMS and our aim of detecting PMS variables will not be largely affected.

\subsubsection{Periods and phase}

The periodicity of a rotating stellar object is generally detectable if solar-like cool spots are present on their surface. However, observable cool spots on PMS star surface is difficult due to their surrounding material. Several authors have reported periodic variability of PMS stars (see Dutta et al. 2018a and the references therein), which generally arises from disk-asymmetry or hot spot (see section 1).

To estimate the period from our time series data, we use the Lomb-Scargle (LS) periodogram analysis technique (Lomb 1976; Scargle 1982), which is the well established method to search for periods even with irregularly-sampled observations. This method first estimates the normalized power for a given frequency and finds the most significant peak (or highest peak) from the periodogram. This peak corresponds to the period of the time series signal. Observed light curves are then folded with this estimated period to obtain phased light curves. Two phased light curves are displayed in Figure 2, with periods of 0.546 (ID364) and 2.309 (ID55) days, which are estimated from the observed data.

Lada, C. J., Lada, E. A., 2003, MNRAS, 41, 57 


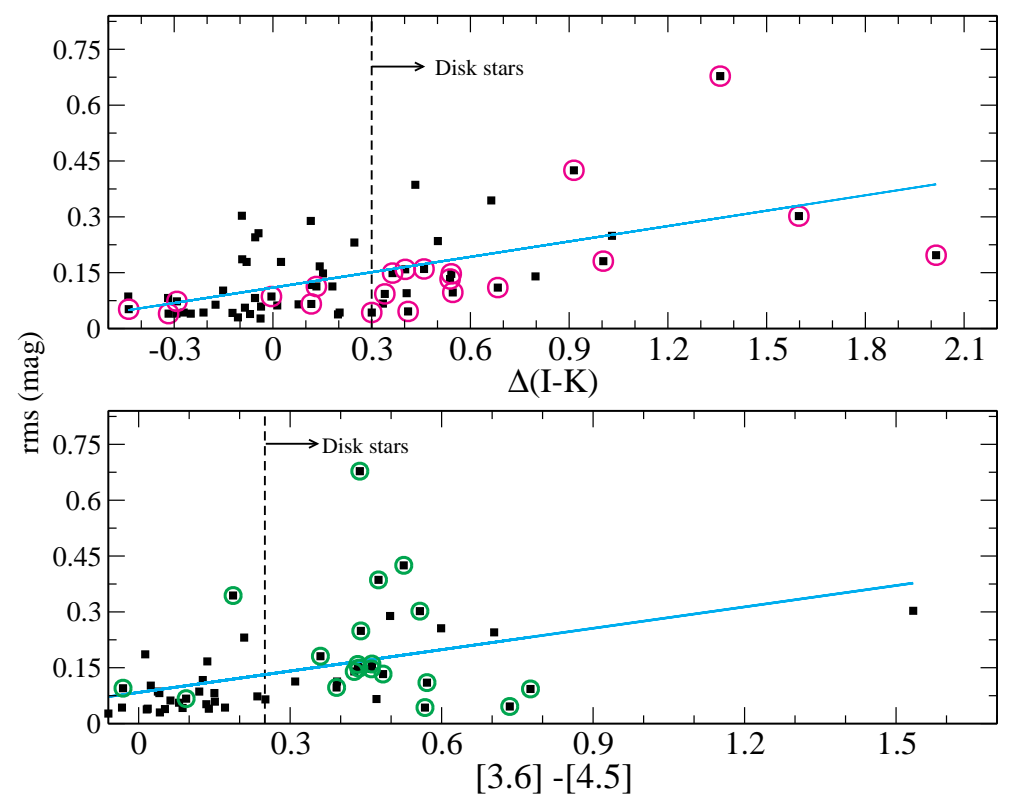

Figure 3: The rms of light curves, which is basically proportional to variability amplitude, is displayed as a function of NIR excess $\Delta(I-K)$ in the upper panel and MIR excess ([3.6]-[4.5]) in the lower panel for periodic as well as aperiodic variables. The vertical dashed lines on both panels are adopted as the boundary between diskless and disk stars. The sources detected as $\mathrm{H} \alpha$ emitters are marked with magenta crosses in the upper panel. In the lower panel, NIR excess sources (see section 4.1) are marked by the green circles. The cyan lines are drawn to sense the increasing trend of rms with infrared disk excess.

\section{Discussions}

\subsection{Detection of surrounding disk}

The presence of circumstellar material defines a star as being young ( $<10$ Myrs). Spectroscopy is generally considered as the most robust technique to identify the nature of stellar objects. However taking account of the time required by spectroscopy and telescope availability, infrared excess is advantageously used to detect young stars in large samples (Lada 1987, Evans et al. 2009, Dutta et al. 2018b, Dutta et al. 2019).

Hillenbrand et al. (1998) pointed out that disk-bearing sources could be identified from excess in $(\mathrm{I}-\mathrm{K})$ with respect to their intrinsic colours. Following Rodríguez-Ledesma et al. (2010), we have estimated intrinsic colours of the variable sources. Thus, stars with $\Delta(I-K)>0.3$ mag are defined as disk-bearing stars (Herbst et al. 2002; Makidon et al. 2004). We have also noticed that the Spitzer-IRAC colour [3.6] - [4.5] is greater than 0.25 mag for infrared excess sources (green circles in the lower panel of Figure 3), which is slightly greater than 0.15 mag as considered by Gutermuth et al. (2009). We have also identified the NIR excess sources from $J H K$ observations of UKIDSS survey, CFHT-WIRCAM, some of them also located at [3.6] - [4.5] $<0.25$ mag. Since $\mathrm{H} \alpha$ emission is an important tracer of young stellar activity, we make use of IPHAS photometry to obtain the $\mathrm{H} \alpha$ emission sources (magenta circle in the upper panel of Figure 3).

From our analyses, we found that around $65 \%$ of identified variables show either infrared excess or $\mathrm{H} \alpha$ emission in our studied regions. Thus, circumstellar material could have a strong contribution to the variability in those sample. In addition, variability could be used to identify membership in those young clusters. 


\subsection{Contribution of the circumstellar disk on stellar variability}

A careful inspection of Figure 3, we notice that a fraction of disk-bearing stars have larger amplitude (analogous to relatively higher $\mathrm{rms}$ ) of variation. Such an increasing trend of amplitude with infrared access might be an indication of the presence of accreting gas around the inner disk, which contributes to the photometric variability (Cody et al. 2018). We suggest that the variability of those young stars is possibly originating from star-disk interaction, asymmetric disk obscuration, accretion outbursts or any other structural modification in the inner disk regions. However, from our limited studies, such investigation is inconclusive.

\section{Summary}

In this paper, we have extended our work on time series photometric analysis of a few young clusters. Our optical $I$-band observations help to identify variable stars of those regions. Analysis of the observed dataset reveals that nearly $65 \%$ of disk-bearing members of NGC 2282 are photometrically variable.

We have explored the correlation between circumstellar disk and variability using the amplitudes of variation and infrared excess. We find that the younger objects have larger amplitudes of variation. We suggest that the variability of those young stars are largely contributed from the circumstellar material. Further tests are needed to explore the framework of variability classification and related physical mechanisms.

\section{References}

Barentsen et al. 2014, MNRAS, 444, 3257

Cieza L., Baliber N. 2007, ApJ, 671, 605

Costa V. M., Lago M. T. V. T., Norci L. et al. 2000, A\&A, 354, 621

Cody A. M., Hillenbrand L. A. 2018, AJ, 156, 71

Dahm S. E. 2008, in Handbook of Star Forming Regions, Volume I, ed. B. Reipurth, 966

Dutta S., Mondal S., Jose J. et al. 2015, MNRAS, 454, 3597

Dutta S., Mondal S., Joshi S. et al. 2018a, MNRAS, 476, 2813

Dutta S., Mondal S., Samal M. R. et al. 2018b, ApJ, 864, 154

Dutta S., Mondal S., Joshi S. et al. 2019, MNRAS, 487, 1765

Evans II N. J., Dunham M. M., Jorgensen J. K. et al. 2009, ApJS, 181, 321

Favata F., Flaccomio E., Reale F. et al. 2005, ApJS, 160, 469

Gutermuth R. A., Megeath S. T., Myers P. C. et al. 2009, ApJS, 184, 18

Herbst W., Herbst D. K., Grossman E. J. et al. 1994, AJ, 108, 1906

Herbst W., Bailer-Jones C. A. L., Mundt R. et al. 2002, A\&A, 396, 513

Herbst W., Eislöffel J., Mundt R. et al. 2007, Protostars and Planets V, 297

Hillenbrand L. A., Strom S. E., Calvet N. et al. 1998, AJ, 116, 1816

Irwin J., Hodgkin S., Aigrain S. et al. 2008, MNRAS, 384, 675

Joy A. H. 1945, ApJ, 102, 168

Kowalski A. F., Hawley S. L., Holtzman J. A. et al. 2010, ApJ, 714, L98

Lada C. J. 1987, in Proc. IAU Symp. 115, Star Forming Regions, ed. M. Piembert \& J. Jugaku (Dordrecht: Reidel), 1

Lada C. J., Lada E. A., 2003, MNRAS, 41, 57

Lomb N. R. 1976, Ap\&SS, 39, 447

Lucas P. W., Hoare M. G., Longmore A. et al. 2008, MNRAS, 391, 136

Mahdavi A., Kenyon S. J. 1998, ApJ, 497, 342

Makidon R. B., Rebull L. M., Strom S. E. et al. 2004, AJ, 127, 2228

Rebull L. M., Stauffer J. R., Megeath S. T. et al. 2006, ApJ, 646, 297

Reipurth B., Schneider N. 2008, Star Formation and Young Clusters in Cygnus. p. 36 
Rodríguez-Ledesma M. V., Mundt R., Eislffel J. 2010, A\&A, 515, A13

Romanova M. M., Ustyugova G. V., Koldoba, A. V. et al. 2004, ApJ, 616, L151,

Scargle J. D. 1982, ApJ, 263, 835

Turner N. J., Carballido A., Sano T. 2010, ApJ, 708, 188

Vorobyov E. I., Basu S. 2010, ApJ, 719, 1896 